\title{
LE DESS DE BIOLOGIE DE LA REPRODUCTION
}

La formation est mise en place à partir du 1er Octobre 1996 dans le cadre de l'Université Paris V.

L'enseignement théorique et pratique, centralisé au plan national sera organisé en 4 semaines de 5 jours $1 / 2$ (une tous les deux mois, environ). Une partie de l'enseignement sera assurée dans les différentes universités sous la responsabilité des membres du comité de direction du diplôme.

\section{Responsable de la formation}

Professeur Pierre JOUANNET

Laboratoire d'Histologie-Embryologie - Biologie de la Reproduction

Groupe Hospitalier Cochin

123 Bd Port Royal

75014 PARIS

\section{Comité de Direction}

$\begin{array}{ll}\text { Pr. J.F.GUERIN } & \text {-Lyon } \\ \text { Pr. C.HUMEAU } & \text {-Montpellier } \\ \text { Pr. D.LE LANNOU } & \text {-Rennes } \\ \text { Dr. J.MANDELBAUM } & \text {-INSERM - Hôpital Necker Paris } \\ \text { Pr. B.SELE } & \text {-Grenoble } \\ \text { Pr. F.THEPOT } & \text {-Amiens }\end{array}$

\section{Modalités d'accès}

Sélection sur dossier et/ou audition des candidats .

Conditions d'admission :

-Interne ou ancien interne de Biologie Médicale en Médecine ou en Pharmacie ayant validé un certificat de Biologie de la Reproduction en MSBM (*)

-Scientifique ayant validé une maîtrise de Sciences de la Vie.

(*) Les certificats de Biologie de la Reproduction, actuellement enseignés dans de nombreux CHU, disparaîtront progressivement au cours des 3 prochaines années scolaires. De nouvelles modalités d'accès au DESS seront donc définies ultérieurement. 


\section{Programme de l'enseignement}

\section{MODULE 1 : Appareil génital mâle et exploration de l'infertilité masculine}

Enseignement théorique (30h)

-La spermatogenèse

-Les glandes annexes et la fonction du liquide séminal

-Maturation épididymaire et capacitation des spermatozoïdes

-Régulations

-Pathologies

\section{Enseignement pratique}

-deux modules obligatoires

\section{1-Analyse du sperme ( 5 jours)}

Spermogramme, spermocytogramme, tests fonctionnels (test de Hühner et test de pénétration croisé), MAR test, expression et analyse des résultats.

2-Techniques de préparation du sperme pour insémination artificielle et fécondation in vitro (2 jours)

Centrifugation-migration, migration ascendante, migration sur Percoll, isolement des spermatozoïdes à partir des urines (éjaculation rétrograde).

-deux modules optionnels au choix parmi les suivants (chaque module aura une durée de 2 jours) :

1-Mesure des marqueurs biochimiques du plasma séminal

2-Mise en évidence et quantification des anticorps antispermatozoïdes

3-Analyse du mouvement des spermatozoïdes par vidéo assistée par ordinateur

4-Etude de la fonction acrosomique (anticorps monoclonaux, Lectine)

5-Etude de l'interaction gamétique (spermatozoïde-zone pellucide, spermatozoïde-ovocyte)

6-Etude des spermatozoïdes en microscopie électronique

7-Analyse des biopsies testiculaires

8-Etude bactériologique du sperme.

MODULE 2 : Traitement des ovocytes et fécondation in vitro

\section{Enseignement théorique (10h)}

-Ovo et folliculogenèse

-Maturation ovocytaire

-Fécondation

-Régulation

-Pathologie

\section{Enseignement pratique}

3 modules obligatoires

1- Fécondation in vitro (5 jours)

-Détection et analyse des complexes cumulo-ovocytaires

-Fécondation in vitro (techniques en puits et en microgoutte,...) 
-GIFT

-Préparation des catheters pour le transfert embryonnaire.

2-FIV avec micromanipulation (4 jours)

-Fabrication des micropipettes

-Techniques de microinjection

3-Culture et coculture des embryons jusqu'au stade blastocyste

MODULE 3 : Congélation des gamètes - Conservation des gamètes et des embryons - Génétique des gamètes et des embryons.

\section{Enseignement théorique (40h)}

-Principe de la congélation cellulaire

-Anomalies génétiques de la fécondation

-Développement embryonnaire pré-implantatoire

\section{Enseignement pratique}

3. Modules obligatoires

1-Congélation des spermatozoïdes $(1,5 j o u r)$

2- Congélation des embryons (1,5jour)

3-Analyse génétique des gamètes et des embryons. Caryotype.Hybridation in situ. PcR (5jours)

\section{MODULE 4 : Aspects méthodologiques, réglementaires et éthiques}

\section{Enseignement théorique (40h)}

-Modalités d'analyse et d'évaluation des résultats

-Conduite d'un essai clinique

-Contrôle de qualité : principes et modalités

-Test de toxicité du matériel et des milieux

-Don de gamètes et d'embryons

-Recrutement des donneurs et risque sanitaire

-Recrutement des donneurs et risque héréditaire

-Critère de choix des données pour les couples receveurs

-Organisation et gestion des centres conservant des gamètes et des embryons

-Aspects législatifs et réglementaires des actes biologiques de l'assistance médicales à la procrétaion

-Questions éthiques suscitées par les actes de l'AMP

\section{Enseignement dirigé (20h)}

-Organisation d'un contrôle de qualité

-Modalité d'expression des résultats

-Analyse de dossiers

-Analyses d'articles 


\section{Anglais et informatique}

Une série d'articles en langue anglaise devra être analysée pour la connaissance des protocoles techniques utilisés. La rédaction du mémoire s'appuiera sur une analyse bibliographique faite en majorité à partir d'articles en langue anglaise.

Les laboratoires d'accueil étant généralement informatisés les étudiants apprendront l'utilisation de logiciels spécifiques dans la pratique des laboratoires. Une formation spécifique et pratique sera organisée à l'utilisation de l'informatique pour analyser et évaluer les résultats des actes biologiques réalisés.

\section{Stages}

Les stages d'une durée de 6 mois seront organisés dans des laboratoires hospitaliers d'accueil maîtrisant les différentes techniques de Biologie de la Reproduction faisant l'objet de la formation.

L'organisation et le suivi pédagogique des stages seront assurés par les responsables des laboratoires hospitaliers concernés, sur la base d'objectifs définis en liaison avec le comité de direction du DESS.

Le stage sera validé par une appréciation portée par le responsable de laboratoire.

\section{Terrains de stages préssentis}

-Ile de France :

-Cochin (P.JOUANNET)

-Tenon (J.P.DADOUNE, J.MANDELBAUM)

-Necker (M.O.ALNOT, J.MANDELBAUM, M.PLACHOT)

-Pitié (GONZALES)

-Bondy (N...)

-A.Béclère (J.SELVA)

-Poissy (J.P.BISSON, C.ROUSSEL)

-Amiens (F.THEPOT)

-Lille (J.LEONARDELLI, P.SAINT-POL)

-Reims (J.J.ADNET)

-Nancy (B.FOLIGUET, H.GERARD)

-Strasbourg (P.GERLINGER, A.CLAVERT)

-Besançon (J.L.BRESSON)

-Lyon (J.C.CZYBA, J.F.GUERIN)

-Grenoble (B.SELE, P.JALBERT)

-Marseille (J.M.LUCIANI, J.M.GRILLO)

-Nice (P.FENICHEL, M.DONZEAU)

-Montpellier (C.HUMEAU, F.ARNAL)

-Toulouse (J.PARINAUD, A.MANSAT)

-Bordeaux (G.MAYER, C.MATHIEU)

-Clermont-Ferrand (D.BOUCHER, L.JANNY)

-Tours (D.ROYERE, S.HAMAMAH)

-Nantes (P.BARRIERE)

-Rennes (D.LE LANNOU)

-Rouen (B.MACE) 
Le contrôle des connaissances s'effectuera sous forme continue et terminale :

-Contrôle continu au cours du stage (note sur 20)

-Examen écrit (note sur 20)

-Soutenance d'un mémoire (note sur 20)

La moyenne est exigée dans les différentes formes de contrôle

\section{Formation continue}

L'organisation de l'enseignement en modules permettra d'accueillir les personnes intéressées par une formation continue dans les différents domaines concernés. Cette formation peut s'adresser aux Biologistes, Médecins ou Pharmaciens ayant déjà des fonctions dans le secteur, mais aussi aux techniciens. Le nombre d'inscrits sera limité en raison du caractère prioritaire de la formation initiale dans le cadre de ce DESS. 